\title{
Rostral ventrolateral medullary but not medullary lateral tegmental field neurons mediate sympatho-sympathetic reflexes in cats
}

\author{
Susan M. Barman ${ }^{\mathbf{1}}$ and Hakan S. Orer ${ }^{1,2}$ \\ ${ }^{1}$ Department of Pharmacology and Toxicology, Michigan State University, East Lansing, Michigan; and ${ }^{2}$ Department of \\ Pharmacology, Faculty of Medicine, Hacettepe University, Ankara, Turkey
}

Submitted 28 June 2010; accepted in final form 30 August 2010

\begin{abstract}
Barman SM, Orer HS. Rostral ventrolateral medullary but not medullary lateral tegmental field neurons mediate sympatho-sympathetic reflexes in cats. Am J Physiol Regul Integr Comp Physiol 299: R1269-R1278, 2010. First published September 1, 2010; doi:10.1152/ajpregu.00422.2010.-This study was designed to build on past work from this laboratory by testing the hypothesis that medullary lateral tegmental field (LTF) neurons play a critical role in mediating sympathoexcitatory responses to activation of sympathetic afferent fibers. We studied the effects of microinjection of $N$-methylD-aspartate (NMDA) or non-NMDA receptor antagonists or muscimol bilaterally into the LTF on the area under the curve of the computeraveraged sympathoexcitatory potential in the right inferior cardiac nerve elicited by short trains of stimuli applied to afferent fibers in the left inferior cardiac or left splanchnic nerve $(\mathrm{CN}, \mathrm{SN})$ of baroreceptordenervated and vagotomized cats anesthetized with a mixture of diallylbarbiturate and urethane. In contrast to our hypothesis, sympathoexcitatory responses to stimulation of $\mathrm{CN}(n=5-7)$ or $\mathrm{SN}(n=$ 4-7) afferent fibers were not significantly affected by these procedures. We then determined whether the rostral and caudal ventrolateral medulla (RVLM, CVLM) and nucleus tractus solitarius (NTS) were involved in mediating these reflexes. Blockade of non-NMDA, but not NMDA, receptors in the RVLM significantly reduced the area under the curve of the sympathoexcitatory responses to electrical stimulation of either $\mathrm{CN}(P=0.0110 ; n=6)$ or $\mathrm{SN}(P=0.0131$; $n=5)$ afferent fibers. Neither blockade of excitatory amino acid receptors nor chemical inactivation of CVLM or NTS significantly affected the responses. These data show that activation of non-NMDA receptors in the RVLM is a critical step in mediating the sympathosympathetic reflex.
\end{abstract}

cardiac sympathetic afferents; caudal ventrolateral medulla; nucleus tractus solitarius; splanchnic afferents; sympathetic nerve discharge

WORK FROM THIS LABORATORY $(5,7,10,11,31,32,35)$ in the past decade or so has provided evidence that medullary lateral tegmental field (LTF) neurons play a crucial role in setting the resting pattern of sympathetic nerve discharge (SND) and in mediating at least some reflex-induced changes in SND in cats. These studies challenge two popular views of central control of cardiovascular function. First, our data dispute the view (14, $21,39)$ that sympathetic tone in most vascular beds originates from neurons within the rostral ventrolateral medulla (RVLM). Microinjection of a non-NMDA ( $N$-methyl-D-aspartate) excitatory amino acid (EAA) receptor antagonist into LTF significantly reduced total power in SND and mean arterial pressure (MAP) to levels comparable to those produced by microinjection of the same drug into RVLM $(7,10)$. Combined with evidence that the axons of LTF-sympathoexcitatory neurons

Address for reprint requests and other correspondence: S. M. Barman, Dept. of Pharmacology and Toxicology, Michigan State Univ., East Lansing, MI 48824 (e-mail:barman@msu.edu). project to the RVLM (4), these data led us to propose that LTF neurons provide excitatory synaptic input to RVLM-spinal sympathoexcitatory neurons and thus function to support resting blood pressure. Second, our work counters the view (14, $21,36)$ that neurons in the nucleus tractus solitarius (NTS), caudal ventrolateral medulla (CVLM), and RVLM are the sole constituents of medullary pathways that mediate baroreceptor, chemoreceptor, and vagal afferent influences on SND. Rather, our data show that LTF neurons are also elements of these reflex pathways. Specifically, microinjection of NMDA receptor antagonists into LTF significantly reduced baroreceptor and Bezold-Jarisch reflex-induced inhibition of SND $(11,31)$. Modulation of SND by lung inflation afferents was also prevented by blockade of NMDA receptors in the LTF (S. M. Barman and G. L. Gebber, unpublished observation) or by chemical inactivation (muscimol microinjection) of this region (35). Sympathoexcitatory responses produced by activation of arterial chemoreceptors and electrical stimulation of cervical vagal afferent fibers were markedly reduced by blockade of non-NMDA receptors in LTF (32). In contrast, sympathoexcitatory responses elicited by stimulation of sciatic or trigeminal nerve afferent fibers, mesencephalic periaqueductal gray, or posterior hypothalamus were not attenuated, and, in fact, in some cases, they were enhanced by microinjection of EAA receptor antagonists or muscimol into the LTF (32).

Those autonomic reflexes that were shown to have a synapse in the LTF have at least two features in common that distinguish them from the responses shown not to require the LTF. One, the pathways mediating the reflexes have their first synapse in the NTS. Two, the afferent fibers involved in the reflexes arise from cardiorespiratory or visceral rather than somatic effector organs. These two facts raise two questions: Is the LTF an element of all autonomic reflex pathways, in which the primary afferent terminates in the NTS? Is the LTF involved in mediating all visceral-sympathetic, but not somatosympathetic reflexes? These questions served as the focus of the current study, in which we sought to identify the pathways that mediate the sympathoexcitatory response to activation of cardiac and splanchnic nerve afferent fibers (sympatho-sympathetic reflexes). We studied the responses to electrical stimulation of afferent fibers in the left inferior cardiac and splanchnic nerves $(\mathrm{CN}, \mathrm{SN})$. Little is known about the essential supraspinal components of the pathways mediating these reflexes. By studying responses elicited by activation of both $\mathrm{CN}$ and SN afferent fibers, we can determine whether the same or different medullary pathways mediate two types of sympathosympathetic reflexes, one initiated by activation of receptors in a cardiovascular organ (i.e., the heart) and the other by activation of receptors in abdominal organs (i.e., gastrointestinal tract and gallbladder). 
The current study was designed to test the hypothesis that LTF neurons play an essential role in mediating sympathoexcitatory responses to activation of sympathetic afferent fibers. The first aim of the study was to test the effects of blockade of EAA receptors or microinjection of muscimol into the LTF on the magnitude of the sympathoexcitatory responses elicited by electrical stimulation of $\mathrm{CN}$ and $\mathrm{SN}$ sympathetic afferent fibers in baroreceptor-denervated and vagotomized cats anesthetized with a mixture of diallylbarbiturate and urethane. The second aim of the study was to determine the role of the RVLM, CVLM, and NTS in mediating sympatho-sympathetic reflexes. The results demonstrate that the RVLM (but not the LTF, CVLM, or NTS) is a major synaptic station within the sympatho-sympathetic reflex arc in the cat.

\section{METHODS}

General procedures. The protocols used in these studies on 25 male cats $(3.4 \pm 0.2 \mathrm{~kg})$ were approved by the Institutional Animal Care and Use Committee of Michigan State University. Cats were anesthetized with an intraperitoneal injection of a mixture of sodium diallylbarbiturate $(60 \mathrm{mg} / \mathrm{kg})$, urethane $(240 \mathrm{mg} / \mathrm{kg})$, and monoethylurea $(240 \mathrm{mg} / \mathrm{kg})$. A femoral artery and femoral vein were cannulated to measure arterial pressure and to administer drugs, respectively. Cats were placed in a stereotaxic apparatus, paralyzed (gallamine triethiodide, $4 \mathrm{mg} / \mathrm{kg}$ iv, initial dose), pneumothoracotomized, and artificially respired with room air. Normocapnia (end-tidal $\mathrm{CO}_{2}$, $4.34 \pm 0.05 \%$ ) was maintained with the parameters of artificial ventilation set at $36.3 \pm 0.9 \mathrm{cc}$ and $20.3 \pm 0.5 \mathrm{cycles} / \mathrm{min}$. Rectal temperature was kept near $38^{\circ} \mathrm{C}$ with a heat lamp. Before neuromuscular blockade, the adequacy of anesthesia was indicated by the absence of a palpebral reflex. When cats were paralyzed, an adequate level of anesthesia was indicated by the inability of noxious stimuli (pinch, heat, surgery) to increase arterial pressure or SND.

Baroreceptor denervation. Stimulation of afferent nerves can change arterial pressure and, thus, the level of baroreceptor nerve activity, which, in turn, might affect the amplitude of the sympathoexcitatory response to stimulation of afferent fibers in the $\mathrm{CN}$ and $\mathrm{SN}$. To eliminate this confounding influence, we completed this study in baroreceptor-denervated and vagotomized cats. The carotid sinus, aortic depressor, and vagus nerves were sectioned bilaterally. Two observations verified the completeness of baroreceptor denervation. First, spectral analysis failed to show a cardiac related rhythm in SND. Specifically, there was no sharp peak in the autospectrum of SND at the frequency of the heart beat, and coherence values relating SND to the arterial pulse were $<0.1$ at this frequency. Second, SND was not reflexly inhibited during the pressor response produced by an injection of norepinephrine bitartrate (1-2 $\mu \mathrm{g} / \mathrm{kg}$ iv).

Preparation of sympathetic nerves for recording and electrical stimulation. The vertebral portions of the first and/or second rib on the right side was removed to expose the stellate ganglia; the inferior cardiac postganglionic branch of the ganglia was then isolated retropleurally. A monophasic recording was made from the cut central end of this nerve. The preamplifier band pass was set at $1-1,000 \mathrm{~Hz}$, so that the synchronized discharges of sympathetic nerve fibers appeared as slow waves (i.e., envelopes of spikes).

The left $\mathrm{CN}$ was isolated by using the same approach as just described, and the left $\mathrm{SN}$ was isolated via a retroperitoneal approach. The cut central ends of these nerves were placed on bipolar electrodes that were connected to constant current stimulus isolation units and a digital stimulator (Grass Instrument, models nos. PSIU6 and S8800). Short trains (11-ms train duration) of three pulses $(200 \mathrm{~Hz})$ were applied once every $2 \mathrm{~s}$ to the peripheral nerves. This is a common method used to induce sympatho-sympathetic (or somato-sympathetic) reflexes $(32,38,40)$. The stimulus intensity ranged from 0.3 to $1.0 \mathrm{~mA}$, and the square-wave pulse duration was $1.0 \mathrm{~ms}$; in each case, the stimulus intensity was at least three times the threshold for eliciting a sympathoexcitatory response. In most cases, the responses to stimulation of both the $\mathrm{CN}$ and $\mathrm{SN}$ were evaluated. In three preliminary experiments, only the $\mathrm{SN}$ was stimulated, and responses to both left and right inferior cardiac SND were recorded. Stimuli elicited comparable responses in the two nerves, and the effects of microinjection of drugs into the brain stem were the same for the two nerves. Thus, in subsequent experiments, we recorded only right inferior cardiac SND, allowing for stimulation of afferent fibers in the left CN. At the start of most experiments, we also applied highfrequency $(40-\mathrm{Hz})$ stimuli to afferent fibers in the $\mathrm{CN}$ and $\mathrm{SN}$ for 10-15 s, while recording arterial pressure. Stimulus intensity and pulse duration were the same as those used for the short trains of stimuli.

Microinjections. The sites of microinjection into the medulla, as well as the concentrations of EAA receptor antagonists and muscimol, were similar to those used by us in earlier studies $(5,7,9-11,31,32$, $35)$. Drugs were microinjected through a glass micropipette $(\sim 40-\mu \mathrm{m}$ tip diameter) that was glued to the needle of a 5- $\mu$ l Hamilton syringe. The micropipette was filled with a $1.0-\mathrm{mM}$ solution of $1,2,3,4$ tetrahydro-6-nitro-2,3-dioxobenzo-[f]quinoxaline-7-sulfonamide (NBQX disodium salt, a selective non-NMDA receptor antagonist), a 3-mM solution of $\mathrm{D}(-)$-2-amino-5-phosphonopentanoic acid D-AP5 (a selective NMDA receptor antagonist), or a $10-\mathrm{mM}$ solution of muscimol (a GABA receptor agonist). Drugs were diluted in $0.9 \%$ saline; the solution was adjusted to a $\mathrm{pH}$ of 6-8 (litmus paper test). The syringe and micropipette were mounted on a microinjection unit (David Kopf Instruments, model no. 5000). A 100-nl injection of NBQX (100 pmol), D-AP5 (300 pmol), or muscimol (1 nmol), was made slowly $(\sim 20 \mathrm{~s})$ at each medullary site by turning the calibrated micrometer on the microinjection unit. Vehicle ( $0.9 \%$ saline) was also injected into the LTF and RVLM of three cats.

The dorsal surface of the medulla was exposed by removing portions of the occipital bone and cerebellum. The midline, obex, and dorsal medullary surface were used as landmarks for placement of the micropipette in LTF, RVLM, CVLM, or NTS. All injections were made bilaterally. We targeted regions of the LTF, RVLM, and CVLM, where we have identified neurons whose naturally occurring activity is correlated to SND in baroreceptor-innervated and -denervated cats $(3-6,9)$. We targeted the region of the NTS where pulse-synchronous neuronal activity can be recorded in baroreceptorinnervated cats (29). The micropipette was positioned into LTF 2 and $3 \mathrm{~mm}$ rostral to the obex and $2.8 \mathrm{~mm}$ lateral to the midline; microinjections were made at depths of 3 and $4.5 \mathrm{~mm}$ from the dorsal surface. The micropipette was positioned into RVLM 4.5 and $5.5 \mathrm{~mm}$ rostral to the obex and $3.5 \mathrm{~mm}$ lateral to the midline; microinjections were made 4.5 and $5.5 \mathrm{~mm}$ from the dorsal surface. The micropipette was positioned into CVLM 1 and $2.5 \mathrm{~mm}$ rostral to the obex and $4 \mathrm{~mm}$ lateral to the midline; microinjections were made 4.5 and $5.5 \mathrm{~mm}$ from the dorsal surface. The micropipette was positioned into the NTS $1.0 \mathrm{~mm}$ rostral to the obex and $1.6 \mathrm{~mm}$ lateral to the midline, at the level of the obex and $1.4 \mathrm{~mm}$ lateral to the midline, and $1.0 \mathrm{~mm}$ caudal to the obex and $1 \mathrm{~mm}$ lateral to the midline. Microinjections were made at depths of $\sim 1.1 \mathrm{~mm}$ from the dorsal surface in each track. The target sites for the injections are marked by asterisks (*) in the schematics of medullary cross sections in Fig. $1 A$.

To reduce the number of animals needed to complete this project, in most cats, the same or a different drug was injected into two or more medullary regions. Additional injections were made a minimum of $30 \mathrm{~min}$ after one set of injections and only after allowing time for recovery from the effects (if any) of microinjection of a drug into an area on the sympathoexcitatory responses. NBQX was microinjected into both the LTF and RVLM of four cats and into both the CVLM and NTS of five other cats. D-AP5 was microinjected into both the LTF and RVLM of four cats, into both the LTF and CVLM in another cat, and into both the CVLM and NTS of two cats. Muscimol was microinjected into both the LTF and RVLM of one cat, into the 

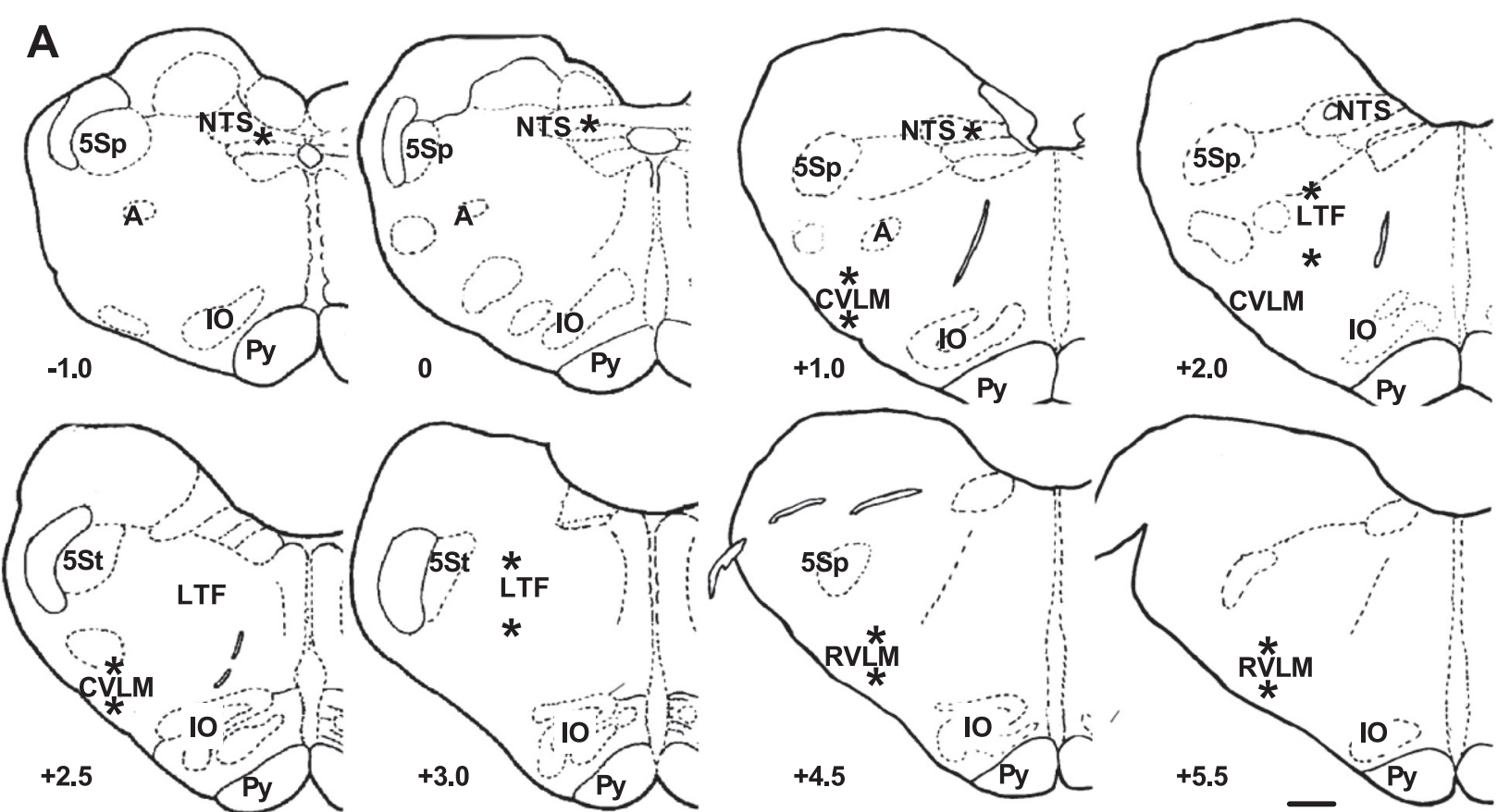

B

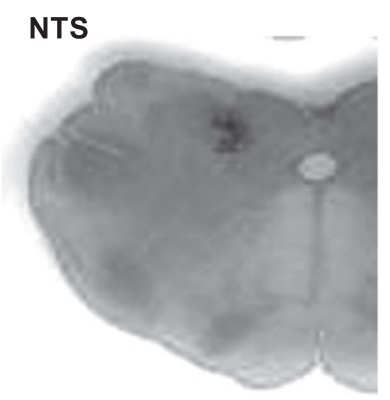

CVLM

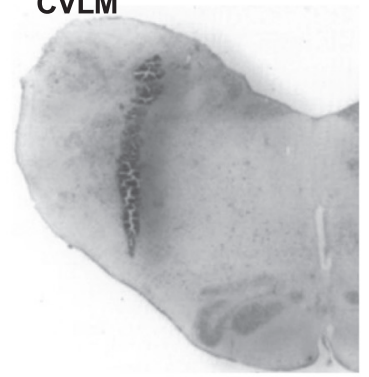

LTF

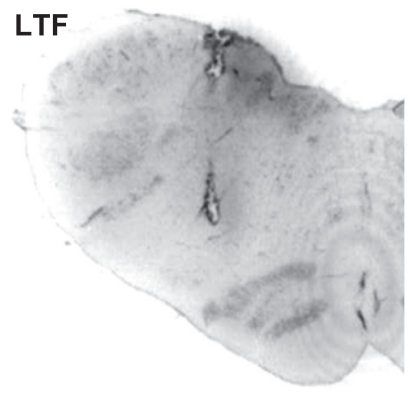

RVLM

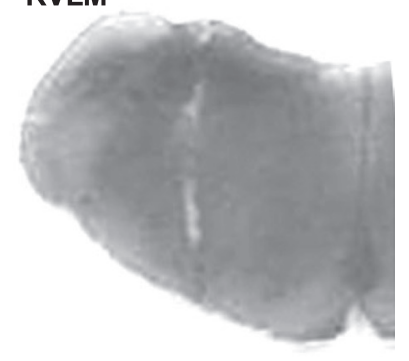

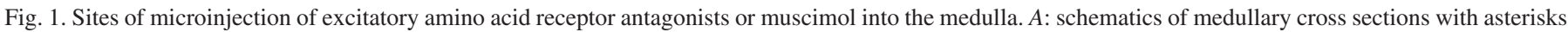

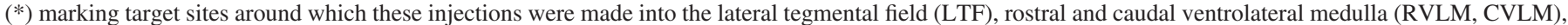

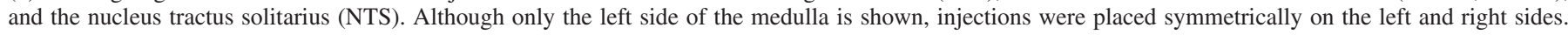

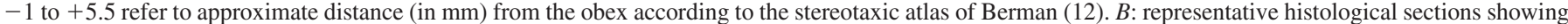

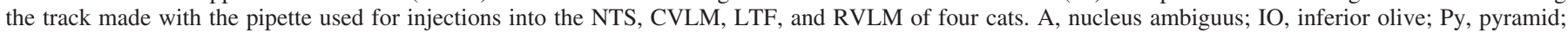
5Sp and 5St, spinal trigeminal nucleus and tract. Scale bar: $1 \mathrm{~mm}$.

CVLM, NTS, and RVLM of two cats, and into both the CVLM and NTS of another cat. The order of injections was random with the exception that microinjection of muscimol into the RVLM was always a final injection. In some cases, a second drug was injected into the same medullary region after waiting at least $1 \mathrm{~h}$. Both NBQX and D-AP5 were microinjected into the LTF of one cat, into the RVLM of two cats, into the CVLM of two cats, and into the NTS of two cats. Both NBQX and muscimol were injected into the CVLM or NTS of two cats. Importantly, the effects of microinjection of a drug into an area were not affected by a previous injection of a drug into the same or a different area.

Data processing. Data were acquired using a Digidata1322A digitizer (Axon Instruments; Union City, CA) at a 200-Hz sampling rate. Datapac software (RUN Technologies; Mission Viejo, CA) was used to construct an average of the sympathetic nerve responses to 45 short trains of stimuli applied to sympathetic afferent fibers in the $\mathrm{CN}$ and $\mathrm{SN}$. ASCII files of the evoked responses were saved for transfer to spreadsheet, graphics, and statistical programs (GraphPad Prism version 5.03 for Windows and GraphPad Instat, GraphPad Software, San Diego CA). A macro written in Microsoft Excel was used to measure the area under the curve of the evoked response (i.e., the area above the dotted horizontal black line in Fig. 2A); this was used as an index of the magnitude of the sympatho-sympathetic reflex.

Fast Fourier transform of SND was performed on 2-min data blocks (47 5-s windows of data with $50 \%$ overlap) with a 5-ms sampling interval. Details of the methods used to construct autospectra of SND can be found in other reports from this laboratory $(8,22)$. In the context of the current study, these data were used primarily to confirm the results of past studies $(5,7,9,10,32)$, in which we quantified the effects of microinjection of these drugs into the medulla on basal SND of baroreceptor-denervated cats.

Experimental protocol. The protocol used in these studies was as follows. We collected control data blocks of responses to trains of stimuli to $\mathrm{CN}$ and/or SN afferent fibers, as well as baseline SND and blood pressure. A second set of control stimulations were collected $\sim 15$ min later to show that the responses were stable. The micropipette was then positioned into one of the medullary regions, and a complete set of injections was made on the left and right sides. Test data blocks were collected 5-10 min after completing the microinjections of a drug into a medullary region. We waited 5-10 min to quantify the effects of these drugs on the sympatho-sympathetic reflex because the maximum changes in basal SND produced by microin- 

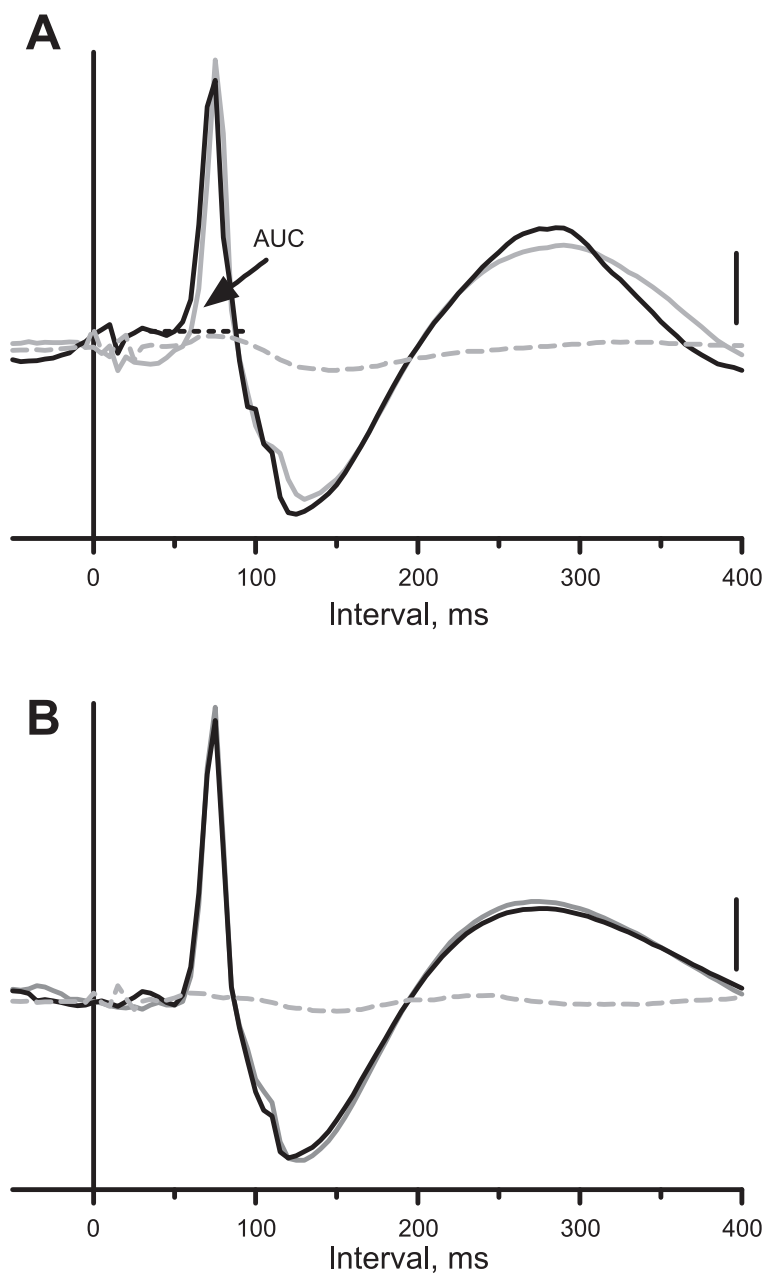

Fig. 2. Evoked responses in the right inferior cardiac nerve produced by electrical stimulation of sympathetic afferent fibers. $A$ : responses to stimulation of the cut central end of the left inferior cardiac nerve before (black trace) and 10 min after (solid gray trace) bilateral microinjection of 1,2,3,4-tetrahydro6-nitro-2,3-dioxobenzo-[f]quinoxaline-7-sulfonamide (NBQX) into the medullary LTF and $5 \mathrm{~min}$ after cervical spinal cord transection (dashed gray trace). $B$ : same as $A$, except stimuli were applied to the cut central end of the left splanchnic nerve. Traces here and in Figs. 4 and 5 show computer-averaged responses in the inferior cardiac nerve to 4511 -ms trains of three pulses (200 $\mathrm{Hz} ; 1.0 \mathrm{~ms}$ ) applied once every $2 \mathrm{~s}$ (applied at time 0 on the $x$-axis). Stimulus intensity was $1.0 \mathrm{~mA}$ in $A$ and $0.3 \mathrm{~mA}$ in $B$. Vertical calibration, $35 \mu \mathrm{V}$ in $A$ and $B$. Area under the curve (AUC) of the evoked response is the area above the dotted horizontal black line.

jection of these drugs occur by this time, and SND reaches a new steady-state level $(7,10,32,35)$. If the drug altered the sympathosympathetic reflex, data were analyzed at $\sim 30$-min intervals for up to $2 \mathrm{~h}$ to allow for partial or full recovery.

At the end of many of the experiments, the spinal cord was transected at an upper cervical level, and the responses to short trains of stimuli to the $\mathrm{CN}$ and $\mathrm{SN}$ were repeated. This was done to determine whether there was a spinal component to the sympathosympathetic reflex $(38-40)$. The brain stem was then removed and fixed in $10 \%$ buffered formalin. Frontal sections $(40-\mu \mathrm{m}$ thickness) were cut and stained with cresyl violet to locate levels of microinjection with reference to the stereotaxic planes of Berman (12). Injection sites were within $\sim 200 \mu \mathrm{m}$ of the target sites described above. Representative histological sections with injection sites in the NTS, CVLM, LTF, and RVLM, are shown in the bottom of Fig. $1 B$.

Statistical analysis. Data are expressed as means \pm SE. Student's paired $t$-test was used to compare the following parameters before and after microinjection of an EAA receptor antagonist or muscimol into the medulla: 1) the area under the curve of the evoked response elicited by short trains of three pulses applied to sympathetic afferent fibers, 2) the onset latency and time to peak of the responses, and 3 ) baseline levels of MAP and total power in SND. A paired $t$-test was also used to compare the changes in MAP elicited by high-frequency stimulation of sympathetic afferent fibers before microinjection of a drug into the medulla. $P<0.05$ indicated statistical significance.

\section{RESULTS}

Characteristics of responses elicited by electrical stimulation of sympathetic afferent fibers. Representative examples of the computer-averaged responses to $4511-\mathrm{ms}$ trains of three pulses applied once every $2 \mathrm{~s}$ to $\mathrm{CN}$ and $\mathrm{SN}$ sympathetic afferent fibers are shown in Fig. 2, $A$ and $B$, respectively. Note that the evoked responses in the right inferior cardiac nerve were triphasic: an initial period of excitation (upward deflection), followed by a period of reduced activity (downward deflection), and then a "rebound" excitation. This study deals with the effects of microinjection of drugs into the medulla on the area under the curve of the initial excitatory response. The onset latency and time to peak of this sympathoexcitatory response in the right inferior cardiac nerve elicited by stimulation of left $\mathrm{CN}$ afferent fibers were $53 \pm 1 \mathrm{~ms}$ and $85 \pm 1 \mathrm{~ms}$ $(n=54)$, respectively. The corresponding values for stimulation of left $\mathrm{SN}$ afferent fibers were $52 \pm 1 \mathrm{~ms}$ and $79 \pm 1 \mathrm{~ms}$ $(n=53)$. These values are the composite data for all control stimulations (before microinjection of a drug into a medullary region) during the course of this study. Except as noted below, these values were not significantly changed by microinjection of drugs into the medulla.

At the start of most experiments, we recorded blood pressure responses to high-frequency $(40 \mathrm{~Hz})$ stimulation of the cut central ends of the $\mathrm{CN}$ and $\mathrm{SN}$ to ensure that the afferent fibers stimulated were involved in mediating cardiovascular responses. We used the same stimulus intensity and duration as used for the short trains of stimuli. High-frequency stimulation of CN and SN significantly $(P<0.0001 ; n=19)$ increased MAP from $99 \pm 4$ to $128 \pm 5 \mathrm{mmHg}$ and from $100 \pm 4$ to $145 \pm$ $7 \mathrm{mmHg}$, respectively.

Effects of microinjection of EAA receptor antagonists or muscimol into LTF on sympatho-sympathetic reflexes. We studied the effects of bilateral microinjection of NBQX into the LTF on the sympathoexcitatory responses in the right inferior cardiac nerve to short trains of stimuli applied to afferent fibers in the left $\mathrm{CN}(n=5)$ or left $\mathrm{SN}(n=4)$. As shown in Fig. 2, $A$ and $B$, the sympathoexcitatory responses were nearly identical before (black traces) and after microinjection of the non-NMDA receptor antagonist (solid gray traces). However, they were eliminated by subsequent cervical spinal cord transection (dashed gray traces). As summarized in Fig. 3A, bilateral microinjection of NBQX into the LTF did not significantly change the area under the curve of the sympathoexcitatory responses elicited by stimulation of either $\mathrm{CN}$ or $\mathrm{SN}$ afferent fibers.

We studied the effects of bilateral microinjection of D-AP5 into the LTF on the sympatho-sympathetic reflex induced by stimulation of $\mathrm{CN}(n=5)$ or $\mathrm{SN}(n=6)$ afferent fibers. Fig. $4 A$ shows an example in which the sympathoexcitatory response to CN stimulation was similar before (solid black trace) and after bilateral microinjection of D-AP5 into the LTF (gray trace). As 

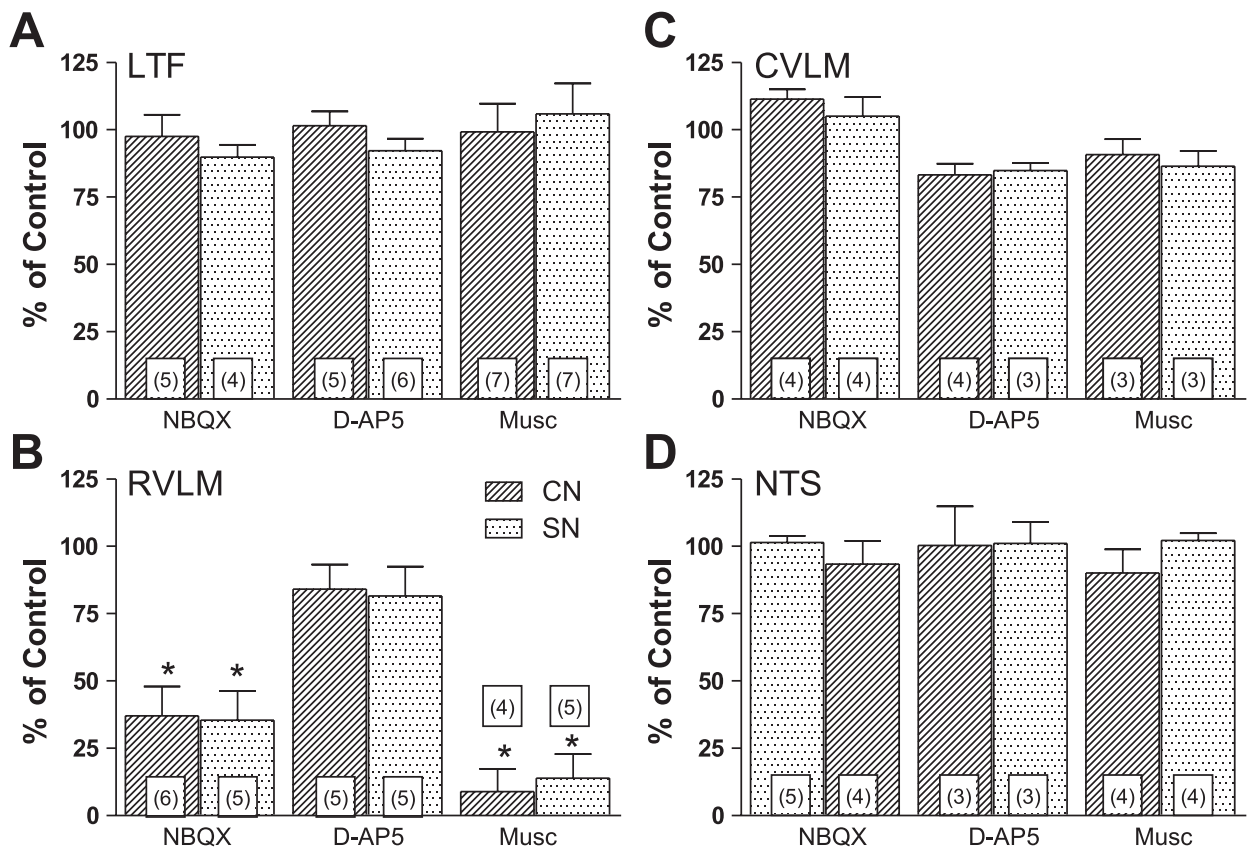

Fig. 3. Summary of effects of bilateral microinjection of drugs into the medulla on the area under the curve of the sympathoexcitatory responses in the right inferior cardiac nerve elicited by stimulation of afferents in the left inferior cardiac $(\mathrm{CN})$ and left splanchnic (SN) nerves. $A$ : changes in the area under the curve of the sympathoexcitatory responses (expressed as percent of control response) elicited by electrical stimulation of afferent fibers in $\mathrm{CN}$ and SN 5-10 min after bilateral microinjection of NBQX, D(-)-2-amino-5-phosphonopentanoic acid (D-AP5), or muscimol (Musc) into the LTF. B-D: same, except microinjections were made into RVLM, CVLM, and NTS, respectively. Data are expressed as means \pm SE. * Statistically different from control. The number in the bars refers to the number of experiments.

summarized in Fig. 3A, the area under the curve of the sympathoexcitatory responses was not significantly affected by microinjection of the NMDA receptor antagonist into the LTF.

As a final test to determine whether LTF neurons contribute to the sympatho-sympathetic reflex, we tested the effects of chemical inactivation of this region on the responses to $\mathrm{CN}$ or SN stimulation. As summarized in Fig. 3A, bilateral microinjection of muscimol into the LTF did not significantly change the area under the curve of the sympathoexcitatory responses to $\mathrm{CN}$ and SN stimulation. An example of the persistence of the sympathoexcitatory response to SN stimulation is shown in Fig. $4 B$; note that the response was eliminated by subsequent cervical spinal cord transection (dashed gray trace). Although not shown, microinjection of vehicle (saline) into the LTF also did not affect the area under the curve of the sympathoexcitatory responses to $\mathrm{CN}(101 \pm 6 \%$ of control, $n=3)$ or SN (98 and $102 \%$ of control; $n=2$ ) stimulation.

Effects of microinjection of EAA receptor antagonists or muscimol into RVLM on sympatho-sympathetic reflexes. Figure $5 A$ shows data from one of the 5 cats, in which the response in the right inferior cardiac nerve to short trains of stimuli applied to the left SN was recorded before (solid black trace) and after bilateral microinjection of NBQX into the RVLM (gray trace). In this example, the area under the curve of the sympathoexcitatory response was reduced to $\sim 25 \%$ of control after the injection; the time to peak of the sympathoexcitatory response was increased from 70 to $85 \mathrm{~ms}$. As was typically the case, the positive potential was reduced in parallel to the excitatory response. The area under the curve of the sympathoexcitatory response was $\sim 88 \%$ of control 45 min after microinjection of NBQX (dashed black trace); the time to peak of the response also recovered.

As summarized in Fig. $3 B$, bilateral microinjection of NBQX into the RVLM significantly reduced the area under the curve of the sympathoexcitatory responses elicited by stimulation of afferent fibers in $\mathrm{CN}(P=0.0110 ; n=6)$ or SN $(P=$ $0.0131 ; n=5)$. Although there was not a significant change in the onset latency of sympathoexcitatory response, the time to peak of the response to stimulation of $\mathrm{CN}$ was significantly increased from $83 \pm 3$ to $95 \pm 5 \mathrm{~ms}(P=0.0042 ; n=5)$. The time to peak of the response to $\mathrm{SN}$ stimulation was also significantly increased from $81 \pm 5$ to $93 \pm 7 \mathrm{~ms}(P=0.0052$; $n=4)$. In one cat, NBQX essentially eliminated the evoked responses; thus, the number of animals is reduced by one for these comparisons.

The NMDA receptor antagonist, D-AP5, was microinjected bilaterally into the RVLM in 5 cats in which sympathosympathetic reflexes were elicited by stimulation of $\mathrm{CN}$ and $\mathrm{SN}$. Figure $5 B$ shows an example of the response to stimulation of $\mathrm{CN}$ afferent fibers before (solid black trace) and after microinjection of D-AP5 (gray trace). Although the area under the curve (97\% of control) was essentially unaffected, the time to peak was delayed by $10 \mathrm{~ms}$. The response was eliminated by subsequent transection of the cervical spinal cord (Fig. 5B, dashed black trace). The effect of microinjection of D-AP5 into the RVLM on the magnitude of the sympathoexcitatory response to $\mathrm{CN}$ and $\mathrm{SN}$ stimulation was variable. The area under the curve of the $\mathrm{CN}$ stimulus-induced response was only $\sim 60 \%$ of control in 2 of 5 cases after microinjection of D-AP5 into the RVLM; likewise, the SN stimulus-induced response was only $55-75 \%$ of control in 3 of 5 cases. However, on a group basis, microinjection of the NMDA receptor antagonist into the RVLM did not significantly affect the magnitude of the evoked responses to $\mathrm{CN}$ or SN stimulation (Fig. $3 B$ ). Nonetheless, there was a modest, but significant $(P=0.0349)$, prolongation in the time to the peak of the sympathoexcitatory response elicited by $\mathrm{CN}$ stimulation from $90 \pm 7$ to $98 \pm 6 \mathrm{~ms}$. This was not the case for the responses to SN stimulation.

Muscimol was microinjected bilaterally into the RVLM in cats in which we stimulated afferent fibers in the $\mathrm{CN}(n=4)$ or SN $(n=5)$. As summarized in Fig. $3 B$, microinjection of the GABA agonist significantly $(P<0.0001)$ decreased the area under the curve of the sympathoexcitatory responses to $\mathrm{CN}$ or $\mathrm{SN}$ stimulation. In fact, the responses were essentially obliter- 

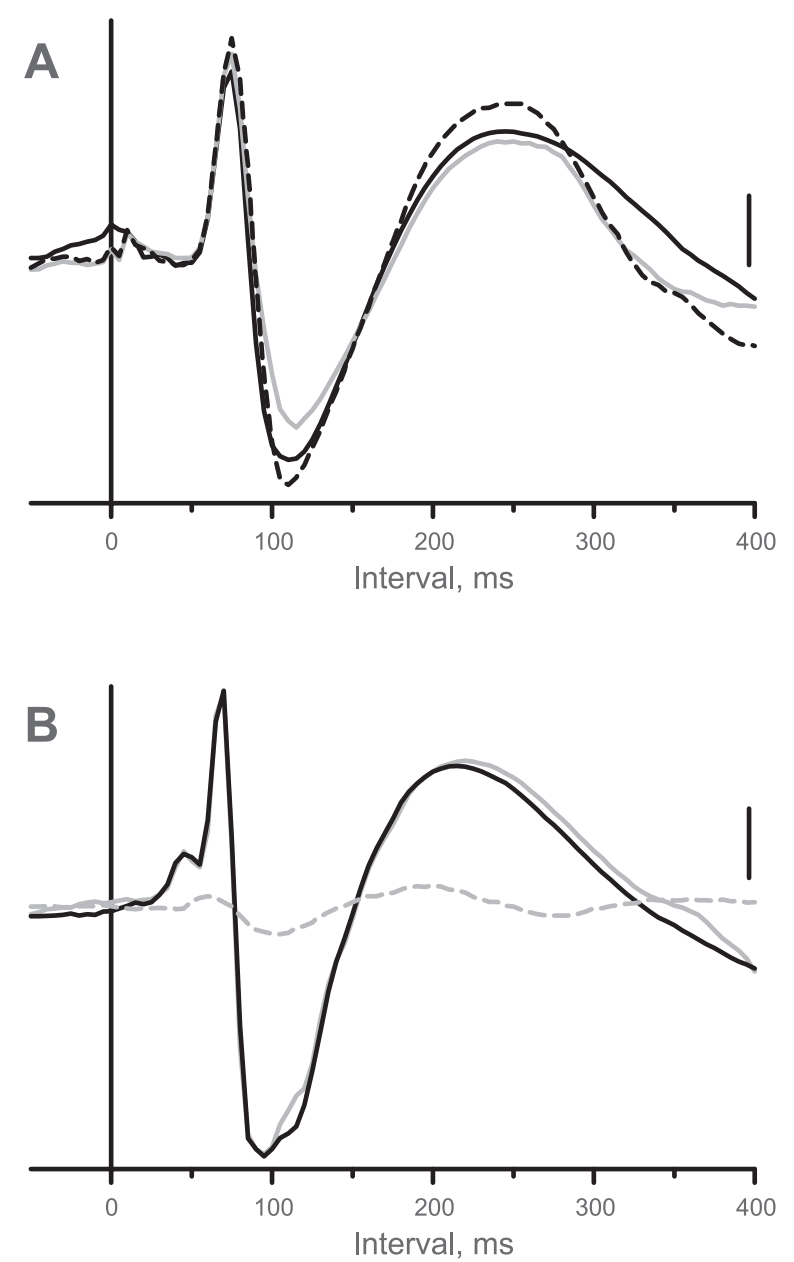

Fig. 4. Examples of sympatho-sympathetic reflexes recorded in the left inferior cardiac nerve before and after bilateral microinjection of drugs into the medulla and cervical spinal cord transection. $A$ : response to stimulation of the left $\mathrm{CN}(0.5 \mathrm{~mA})$ before (solid black trace) and $10 \mathrm{~min}$ after microinjection of D-AP5 into the LTF (gray trace) or the CVLM (dashed black trace). $B$ : response to stimulation of the left $\mathrm{SN}(0.5 \mathrm{~mA})$ before (black trace) and 10 min after microinjection of muscimol into the LTF (gray trace) and 5 min after cervical spinal cord transection (dashed gray trace). Vertical calibration, $50 \mu \mathrm{V}$ (A) and $25 \mu \mathrm{V}(B)$.

ated in most cases. Figure $5 C$ shows data from one of only three experiments in which there was a bimodal peak in the sympathoexcitatory response to stimulation of a sympathetic afferent. Note that the late component of the excitatory response to SN stimulation was abolished by chemical inactivation of RVLM (gray trace), and the early component persisted even after cervical spinal cord transection (dashed gray trace). The spinal component of the response was not included in the assessment of the area under the curve of the response. Although not shown, microinjection of saline into the RVLM of three cats did not affect the area under the curve of the sympathoexcitatory responses to stimulation of $\mathrm{CN}(102 \pm 7 \%$ of control) or SN (97 $\pm 8 \%$ of control).

Effects of microinjection of EAA receptor antagonists or muscimol into CVLM or NTS on sympatho-sympathetic reflexes. As summarized in Fig. 3, $C$ and $D$, microinjection of NBQX, D-AP5, or muscimol into either the CVLM or NTS did not significantly affect the area under the curve of the sympathoexcitatory responses to stimulation of the $\mathrm{CN}$ or $\mathrm{SN}$. Figure
$4 A$ shows the absence of an effect of bilateral microinjection of D-AP5 into the CVLM on the CN stimulus-induced sympathoexcitatory response (dashed black line) in the same cat in which D-AP5 had been injected into the LTF $\sim 30$ min earlier.
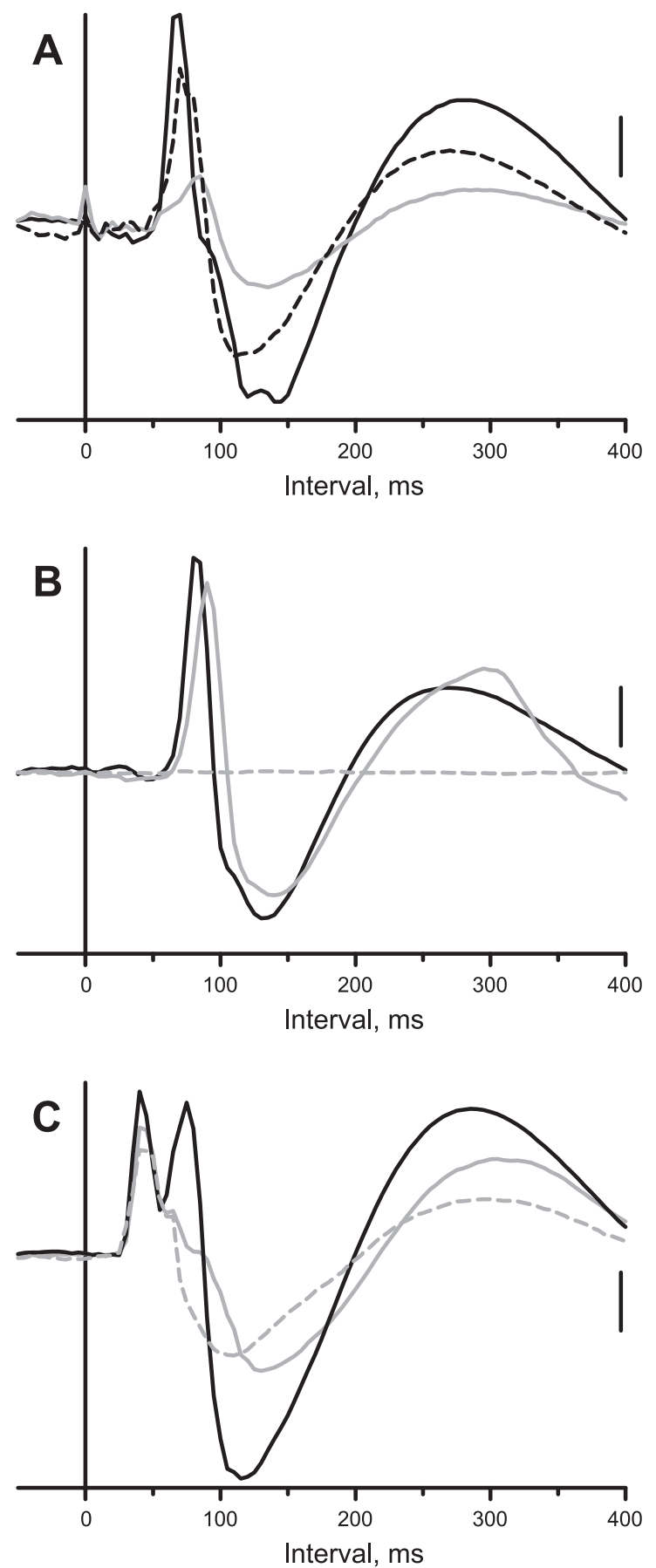

Fig. 5. Effects of microinjection of drugs into the RVLM or cervical spinal cord transection on the sympatho-sympathetic reflex recorded in the right inferior cardiac nerve. $A$ : response to left $\mathrm{SN}$ stimulation $(0.6 \mathrm{~mA})$ before (solid black trace), $10 \mathrm{~min}$ (gray trace), and $45 \mathrm{~min}$ after microinjection of NBQX into RVLM. $B$ : response to left $\mathrm{CN}$ stimulation $(0.6 \mathrm{~mA})$ before (black trace) and after (solid gray trace) microinjection of D-AP5 into RVLM and 5 min after spinal cord transection (dashed gray trace). $C$ : response to left $\mathrm{SN}$ stimulation $(0.5 \mathrm{~mA})$ before (black trace) and after (solid gray trace) microinjection of muscimol into RVLM and 5 min after spinal cord transection (dashed gray trace). Vertical calibration, $55 \mu \mathrm{V}(A), 30 \mu \mathrm{V}(B)$, and $30 \mu \mathrm{V}(C)$. 
Effects of microinjection of EAA receptor antagonists or muscimol on total power in SND and MAP. Table 1 summarizes the effects of bilateral microinjection of EAA receptor antagonists or muscimol into the four medullary regions on MAP and total power in the autospectrum of the right inferior cardiac SND. Microinjection of either NBQX or muscimol but not D-AP5 into the LTF significantly reduced total power in SND and MAP. Microinjection of each of the three drugs into the RVLM or CVLM significantly reduced total power in SND and MAP. Neither SND nor MAP was affected by microinjection of any of the drugs into the NTS. These data corroborate some of the findings from other studies in which EAA receptor antagonists or muscimol were injected into the medulla of baroreceptor-denervated cats $(7,10,32)$. Microinjection of saline into the LTF or RVLM did not affect SND or MAP. Earlier work from his laboratory $(5,7,9-11,31,32,35)$ has also shown that microinjection of saline into the LTF, RVLM, or CVLM does not affect SND or MAP in baroreceptorinnervated or baroreceptor-denervated cats.

\section{DISCUSSION}

The major new finding of the current study is that, among four major medullary nuclei involved in cardiovascular control, only the RVLM is a critical synaptic station within sympatho-sympathetic reflex pathways. This was evidenced by marked attenuation of the area under the curve and a prolongation in the time to peak of the sympathoexcitatory responses to short trains of stimuli applied to afferent fibers in the $\mathrm{CN}$ and

Table 1. Effects of microinjection of NBQX, D-AP5, muscimol, or saline into the medulla on sympathetic nerve discharge and mean arterial pressure

\begin{tabular}{|c|c|c|c|c|}
\hline Injection Site & $n$ & $\begin{array}{c}\text { Total Power in SND, } \\
\% \text { of Control }\end{array}$ & $\begin{array}{c}\text { MAP of Control, } \\
\mathrm{mmHg}\end{array}$ & $\begin{array}{c}\text { MAP After } \\
\text { Injection, mmHg }\end{array}$ \\
\hline \multicolumn{5}{|c|}{$N B Q X, 100 \mathrm{pmol} / 100 \mathrm{nl}$} \\
\hline LTF & 5 & $54 \pm 11^{*}$ & $94 \pm 10$ & $73 \pm 6^{*}$ \\
\hline RVLM & 6 & $46 \pm 6^{*}$ & $92 \pm 7$ & $72 \pm 9 *$ \\
\hline CVLM & 5 & $71 \pm 12 *$ & $106 \pm 12$ & $84 \pm 10^{*}$ \\
\hline NTS & 5 & $105 \pm 5$ & $97 \pm 5$ & $95 \pm 8$ \\
\hline \multicolumn{5}{|c|}{$D-A P 5,300 \mathrm{pmol} / 100 \mathrm{nl}$} \\
\hline LTF & 6 & $79 \pm 11$ & $92 \pm 5$ & $90 \pm 8$ \\
\hline RVLM & 5 & $55 \pm 17 *$ & $99 \pm 5$ & $78 \pm 5^{*}$ \\
\hline CVLM & 3 & $73 \pm 1 *$ & $112 \pm 5$ & $90 \pm 3^{*}$ \\
\hline NTS & 3 & $102 \pm 9$ & $92 \pm 5$ & $95 \pm 5$ \\
\hline \multicolumn{5}{|c|}{ Muscimol, $1 \mathrm{nmol} / 100 \mathrm{nl}$} \\
\hline LTF & 7 & $57 \pm 3 *$ & $109 \pm 6$ & $91 \pm 6 *$ \\
\hline RVLM & 5 & $5 \pm 2 *$ & $96 \pm 9$ & $65 \pm 7 *$ \\
\hline CVLM & 3 & $67 \pm 11 *$ & $87 \pm 9$ & $79 \pm 8 *$ \\
\hline NTS & 4 & $94 \pm 3$ & $95 \pm 8$ & $87 \pm 4$ \\
\hline \multicolumn{5}{|c|}{$0.9 \%$ saline, $100 \mathrm{nl}$} \\
\hline LTF & 3 & $102 \pm 6$ & $115 \pm 4$ & $116 \pm 6$ \\
\hline RVLM & 3 & $104 \pm 8$ & $103 \pm 9$ & $101 \pm 9$ \\
\hline
\end{tabular}

Values are expressed as means \pm SE; $n$, number of experiments. NBQX, 1,2,3,4-tetrahydro-6-nitro-2,3-dioxobenzo-[f]quinoxaline-7-sulfonamide; D-AP5, $\mathrm{D}(-)$-2-amino-5-phosphonopentanoic acid; MAP, mean arterial pressure; LTF, lateral tegmental field; RVLM, rostral ventrolateral medulla; SND, sympathetic nerve discharge; CVLM, caudal ventrolateral medulla; NTS, nucleus of the tractus solitarius. ${ }^{*}$ Significantly different than control $(P \leq$ 0.05 ; paired $t$-test)
SN after bilateral microinjection of NBQX or muscimol into the RVLM of baroreceptor-denervated and vagotomized cats. In contrast, these responses were not significantly affected by microinjection of EAA receptor antagonists or muscimol into the LTF, CVLM, or NTS. These data were counter to our hypothesis that the LTF is involved in mediating sympathosympathetic reflexes.

Certainly, it was not surprising to find that the RVLM is a major synaptic relay station within the sympatho-sympathetic reflex pathways originating from afferents in upper $(\mathrm{CN})$ and middle/lower (SN) spinal thoracic levels. As pointed out by Guyenet (21), neurons in the RVLM "are a nodal point for most, if not all, sympathetic reflexes that involve cardiovascular targets ...." Guo et al. (20) showed that the increases in SND and MAP elicited by epicardial application of bradykinin were significantly attenuated by microinjection of an inhibitor of nitric oxide synthase into the RVLM of cats. To our knowledge, prior to the current study, no one had directly evaluated the role of EAA receptors in the RVLM in mediating the response to activation of afferents in the $\mathrm{CN}$; however, as discussed below, a study by Zhou et al. (45) studied the effects of blockade of EAA receptors in the RVLM on the pressor response to chemical activation of the gallbladder. On the basis of data from the current study, we conclude that sympathosympathetic reflexes initiated by activation of both $\mathrm{CN}$ and $\mathrm{SN}$ afferent fibers are dependent primarily on non-NMDA receptors within the RVLM.

Afferent fibers in the $\mathrm{CN}$ and $\mathrm{SN}$ relay information from both mechanically and chemically sensitive receptors in the heart $(16,28,39)$ and gastrointestinal tract and gallbladder $(15$, $18,39)$, respectively. These afferent fibers contribute to cardiovascular control under at least pathophysiological (e.g., myocardial or abdominal ischemia) conditions $(15,16,18,28)$. Activation (either "natural" or electrical) of these afferent fibers often leads to an increase in efferent sympathetic nerve activity and consequently MAP $(1,17,19,20,23-27,29$, $37-45$ ) in cats and rats. In the current study, we chose to use electrical stimulation of $\mathrm{CN}$ and $\mathrm{SN}$ afferent fibers because it provided a robust and stable sympathoexcitatory response that was easily quantified. We selected parameters of stimulation that elicited a maximal response in the right inferior cardiac nerve and a prominent pressor response during high-frequency stimulation that was comparable to that reported by others who used chemicals (e.g., bradykinin or capsaicin) or distension to activate cardiac, gastrointestinal, or gallbladder receptors (1, $17,19,20,23,25-27,37)$.

The use of computer-averaged evoked responses in sympathetic nerves was popularized beginning in the 1960s for studies of somato-sympathetic (see Ref. 38) and sympatho-sympathetic reflexes (40). Computer averaging diminishes the influence of spontaneous fluctuations in SND. Another advantage of averaging the response to short trains of stimuli separated by $2 \mathrm{~s}$ vs. using continuous high-frequency stimulation is that the evoked response is readily isolated from any stimulus artifact. Also, one can easily separate spinal and supraspinal components of the evoked response based on differences in onset latency and subsequent spinal cord transection. The peak of the evoked response reflects the synchronous activation of the fibers in the sympathetic nerve and thus the synchronous firing of the RVLM spinal nerves. In our experiments, microinjection of NBQX or D-AP5 into the RVLM prolonged the time to the peak of the evoked response to stimu- 
lation of $\mathrm{CN}$ and/or $\mathrm{SN}$ afferent fibers. This implies that these EAA receptor antagonists altered the excitability of RVLM neurons to delay their synchronous firing.

Few studies have entertained the question of which medullary regions contain neurons that are essential elements of sympatho-sympathetic reflex loops. Guo et al. (19) reported that activation of cardiac sympathetic afferent fibers by epicardial application of bradykinin was accompanied by an increase in c-Fos immunoreactivity in NTS, LTF, CVLM, and RVLM neurons in cats. Also, electrical or chemical activation of cardiac sympathetic afferent fibers has been shown to excite neurons in the RVLM $(13,20,24,42,44), \operatorname{CVLM}(13,44)$, NTS $(29,41,43)$, and $\operatorname{LTF}(13,43)$ in cats and rats. Zhou et al. (45) reported that chemical stimulation of the gallbladder increased c-Fos immunoreactivity and glutamate release in the RVLM. Also, electrical stimulation of the $\mathrm{SN}$ has been shown to activate neurons in the $\operatorname{RVLM}(41,45), \operatorname{CVLM}(33,34)$, and NTS (2) in cats and rats. Thus, there was reason to expect that neurons in any one of these areas might contribute to the sympathoexcitatory response elicited by electrical activation of afferent fibers in the $\mathrm{CN}$ or $\mathrm{SN}$.

With the exception of a study by Li et al. (23), in which blockade of non-NMDA receptors in the commissural region of the NTS prevented the increases in renal SND and blood pressure produced by epicardial application of capsaicin in rats anesthetized with a mixture of chloralose and pentobarbital, we are unaware of other studies that tested the effect of disrupting neurotransmission in the medulla on the reflex responses to activation of cardiac sympathetic afferent fibers. We did not specifically target the commissural region of the NTS in this study, as we injected drugs into the region of the NTS where blockade of non-NMDA receptors disrupts the baroreceptor reflex $(31,36)$ and where neurons with pulse-synchronous activity have been identified in baroreceptor-innervated cats (29). Nonetheless, injection volumes of $100 \mathrm{nl} 1 \mathrm{~mm}$ caudal to the obex and $1 \mathrm{~mm}$ lateral to the midline in the current study might be expected to spread to the commissural region of the NTS (30). Our failure to alter the response to electrical stimulation of $\mathrm{CN}$ afferent fibers by microinjection of drugs in the NTS might mean that there is a species difference regarding the role of this region in mediating sympatho-sympathetic reflexes. One might also argue that the use of a mixture of diallylbarbiturate and urethane in our experiments interfered with the role of the NTS in mediating sympatho-sympathetic reflexes. However, several studies $(3,4,11,22,29,35)$ show that this anesthetic regimen does not hinder the ability of NTS to mediate other reflexes (e.g., baroreceptor, Bezold-Jarisch, and lung-inflation).

Zhou et al. (45) reported that unilateral microinjection of either an NMDA or a non-NMDA receptor antagonist into the RVLM nearly abolished the pressor response to application of bradykinin to the serosal surface of the gallbladder in cats. Our data offer some support for their finding in that microinjection of NBQX into the RVLM markedly attenuated the sympathoexcitatory response to electrical stimulation of sympathetic afferents. We also saw a modest but significant delay in the time to peak of the sympathoexcitatory response to stimulation of $\mathrm{CN}$ (but not $\mathrm{SN}$ ) afferent fibers after microinjection of D-AP5 into the RVLM. Although in some cases, the magnitude of the sympathoexcitatory response to $\mathrm{CN}$ or $\mathrm{SN}$ stimulation was attenuated by microinjection of this NMDA receptor antagonist into the RVLM, on a group basis, it was not significantly affected. Thus, we did not identify a significant role for NMDA receptors in the RVLM in mediating sympatho-sympathetic reflexes. NMDA receptors in the RVLM may be critical only for mediating effects due to activation of chemosensitive receptors in the gallbladder. Perhaps this group of afferents accounted for only a small component of the sympathoexcitation elicited by electrical stimulation of the SN. Another difference between our study and that of Zhou et al. (45) may be the location of the microinjections. In contrast to the study by Zhou et al. (45), microinjection of EAA receptor antagonists into the RVLM in our experiments significantly reduced SND and MAP in agreement with earlier work $(7,10)$. We targeted the area of the RVLM where we have extensive experience with the identification of neurons with activity correlated to SND that project directly to the thoracic intermediolateral cell column $(3,4,6)$. Another difference in the two studies is the anesthetic; Zhou et al. (45) used chloralose, and we used a mixture of diallylbarbiturate and urethane. Perhaps the role played by a particular EAA receptor subtype in mediating a reflex can be enhanced or suppressed depending on the type of anesthesia used.

Electrical stimulation of sympathetic afferents have been shown to elicit reflexes that have both spinal and supraspinal components $(38-40)$. Failure to affect the area under the curve of the sympathoexcitatory responses by microinjection of EAA receptor antagonists or muscimol into the LTF, CVLM, or NTS cannot be explained by persistence of a spinal reflex as the sympathoexcitatory responses that we quantified were routinely eliminated by spinal cord transection. Indeed, we only rarely saw a spinal component to the sympatho-sympathetic reflex response in the right inferior cardiac nerve during stimulation of afferent fibers in the left $\mathrm{CN}$ or left $\mathrm{SN}$.

The reduction in the sympathoexcitatory responses to either $\mathrm{CN}$ or $\mathrm{SN}$ after microinjection of $\mathrm{NBQX}$ or muscimol into the RVLM cannot be attributed to the decrease in baseline level of SND as the sympatho-sympathetic reflex responses were unaffected despite marked reductions in baseline levels of SND after microinjection of one or more drugs into the LTF or CVLM or after microinjection of D-AP5 into the RVLM.

As is commonly seen with stimulation of visceral and somatic afferents $(32,38,40)$, short trains of three pulses elicited a stereotypical response that included an initial excitation followed by a period of reduced activity (positive potential). This latter phase is referred to as postexcitatory depression or a "silent period" and signifies that afferent stimulation induces both excitatory and inhibitory influences on the sympathetic nervous system (38). Since microinjection of NBQX into the RVLM reduced the magnitude of the positive potential in parallel to that of the excitatory response, this component of the response primarily may have simply reflected the recovery process of postganglionic neurons following their activation.

\section{Perspectives and Significance}

The original intent of this study was to gain insight into the role of the LTF in mediating excitatory sympatho-sympathetic reflexes. Data from past work from this laboratory $(11,31,32,35)$ raised two questions about the involvement of the LTF in reflex control of SND. Is the LTF an element of all autonomic reflex pathways in which the primary afferent terminates in the NTS? Is 
the LTF involved in mediating all visceral-sympathetic but not somato-sympathetic reflexes? On the basis of the data from the current study, the answer to the first question is a "qualified" "Yes"; the answer to the second question is a definitive "No." The LTF is involved in mediating sympathoexcitatory and sympathoinhibitory responses elicited by natural or electrical activation of afferent fibers in at least two visceral cranial nerves (vagus and glossopharyngeal), which synapse in the NTS $(11,31,32,35)$, but not a somatic cranial nerve (trigeminal), which does not synapse in the NTS (32). The LTF does not mediate reflexes elicited by activation of somatic (sciatic) or visceral $(\mathrm{SN}, \mathrm{CN})$ afferent fibers that synapse in the dorsal horn of the spinal cord (32, current study). It seems that the LTF is an element only within those reflex pathways, in which the first synapse is in the NTS; however, there may be some reflexes that are relayed through the NTS but have not yet been examined as to whether they also have a synapse in the LTF. Another common feature of the visceral reflexes that require the LTF is that they are active under physiological conditions (e.g., baroreceptor, lung inflation, and chemoreceptor reflexes). There is less evidence that $\mathrm{CN}$ and $\mathrm{SN}$ afferent fibers contribute remarkably to setting basal levels of SND and blood pressure.

\section{GRANTS}

This study was supported by National Institutes of Health grant HL33266.

\section{DISCLOSURES}

No conflicts of interest, financial or otherwise, are declared by the authors.

\section{REFERENCES}

1. Ammons WS, Foreman RD. Cardiovascular and T2-T4 dorsal horn cell responses to gallbladder distention in the cat. Brain Res 321: 267-277, 1984.

2. Barber WD, Yuan CS. Gastric vagal-splanchnic interactions in the brainstem of the cat. Brain Res 487: 1-8, 1989.

3. Barman SM, Gebber GL. Axonal projection patterns of ventrolateral medullospinal sympathoexcitatory neurons. J Neurophysiol 53: 15511566, 1985.

4. Barman SM, Gebber GL. Lateral tegmental field neurons of cat medulla: a source of basal activity of ventrolateral medullospinal sympathoexcitatory neurons. J Neurophysiol 57: 1410-1424, 1987.

5. Barman SM, Gebber GL. Lateral tegmental field neurons play a permissive role in governing the $10-\mathrm{Hz}$ rhythm in sympathetic nerve discharge. Am J Physiol Regul Integr Comp Physiol 265: R1006-R1013, 1993.

6. Barman SM, Gebber GL. 'Rapid' rhythmic discharges of sympathetic nerves: Sources, mechanisms of generation, and physiological relevance. J Biol Rhythms 15: 365-379, 2000.

7. Barman SM, Gebber GL, Orer HS. Medullary lateral tegmental field: An important source of basal sympathetic nerve discharge in the cat. Am J Physiol Regul Integr Comp Physiol 278: R995-R1004, 2000.

8. Barman SM, Kenney MJ. Methods of analysis and physiological relevance of rhythms in sympathetic nerve discharge. American Physiological Society Symposium on "Oscillations and Rhythms in the Neural Control of the Circulation." Clin Exp Physiol Pharmacol 34: 350-355, 2007.

9. Barman SM, Orer HS, Gebber GL. Caudal ventrolateral medullary neurons are elements of the network responsible for the $10-\mathrm{Hz}$ rhythm in sympathetic nerve discharge. J Neurophysiol 72: 106-120, 1994.

10. Barman SM, Orer HS, Gebber GL. Role of medullary excitatory amino acid receptors in mediating the $10-\mathrm{Hz}$ rhythm in sympathetic nerve discharge of cats. Brain Res 1049: 249-253, 2005.

11. Barman SM, Phillips SW, Gebber GL. Medullary lateral tegmental field mediates the cardiovascular but not respiratory component of the BezoldJarisch reflex in the cat. Am J Physiol Regul Integr Comp Physiol 289: R1693-R1702, 2005.

12. Berman AL. The Brain Stem of the Cat. A Cytoarchitectonic Atlas with Stereotaxic Coordinates. Madison, WI: University of Wisconsin Press, 1968.
13. Blair RW. Convergence of sympathetic, vagal, and other sensory inputs onto neurons in feline ventrolateral medulla. Am J Physiol Heart Circ Physiol 260: H1918-H1928, 1991.

14. Blessing WW. The Lower Brainstem and Bodily Homeostasis. New York: Oxford University Press, 1997.

15. Cervero F. Sensory innervation of the viscera: Peripheral basis of visceral pain. Physiol Rev 74: 95-138, 1994.

16. Foreman RD. Mechanisms of cardiac pain. Annu Rev Physiol 61: 143-67, 1999.

17. Gao ZL, Zhu Z, Zucker IH, Wang W. Cardiac sympathetic afferent stimulation impairs baroreflex control of renal sympathetic nerve activity in rats. Am J Physiol Heart Circ Physiol 286: H1706-H1711, 2004.

18. Grundy D. Neuroanatomy of visceral nociception: vagal and splanchnic afferent. Gut 51 Suppl I: i2-i5, 2002.

19. Guo ZL, Lai HC, Longhurst JC. Medullary pathways involved in cardiac sympathoexcitatory reflexes in the cat. Brain Res 925: 55-66, 2002.

20. Guo ZL, Tjen-ALooi SC, Li P, Longhurst JC. Nitric oxide in rostral ventrolateral medulla regulates cardiac-sympathetic reflexes: role of synthase isoforms. Am J Physiol Heart Circ Physiol 297: H1478-H1486, 2009.

21. Guyenet PG. The sympathetic control of blood pressure. Nat Rev Neurosci 7: 335-346, 2006.

22. Kocsis B, Gebber GL, Barman SM, Kenney MJ. Relationships between activity of sympathetic nerve pairs: Phase and coherence. Am J Physiol Regul Integr Comp Physiol 259: R549-R560, 1990.

23. Li DP, Averill DB, Pan HL. Differential roles for glutamate receptor subtypes within commissural NTS in cardiac-sympathetic reflex. Am J Physiol Regul Integr Comp Physiol 281: R935-R943, 2001.

24. Li DP, Pan HL. Responses of neurons in rostral ventrolateral medulla to activation of cardiac receptors in rats. Am J Physiol Heart Circ Physiol 279: H2549-H2557, 2000.

25. Li P, Rowshan K, Crisostomo Tjen-ALooi SC, Longhurst JC. Effect of electroacupuncture on pressor reflex during gastric distension. Am J Physiol Regul Integr Comp Physiol 283: R1335-R1345, 2002.

26. Longhurst JC, Kaufman MP, Ordway GA, Musch TI. Effects of bradykinin and capsaicin on endings of afferent fibers from abdominal visceral organs. Am J Physiol Heart Circ Physiol 247: H552-H559, 1984.

27. Longhurst JC, Spilker HL, Ordway GA. Cardiovascular reflexes elicited by passive gastric distension in anesthetized cats. Am J Physiol Heart Circ Physiol 240: H539-H545, 1981.

28. Malliani A, Montano N. Emerging excitatory role of cardiovascular sympathetic afferents in pathophysiological conditions. Hypertension 39: 63-68, 2002.

29. McCall RB, Gebber GL, Barman SM. Spinal interneurons in the baroreceptor reflex arc. Am J Physiol Heart Circ Physiol 232: H657H665, 1977.

30. Nicholson C. Diffusion from an injected volume of a substance in the brain tissue with arbitrary volume fraction and tortuosity. Brain Res 333:325-329, 1985.

31. Orer HS, Barman SM, Gebber GL, Sykes SM. Medullary lateral tegmental field: An important synaptic relay in the baroreceptor reflex pathway of the cat. Am J Physiol Regul Integr Comp Physiol 277: R1462-R1475, 1999.

32. Orer HS, Gebber GL, Phillips SW, Barman SM. Role of the medullary lateral tegmental field in reflex-mediated sympathoexcitation in cats. Am J Physiol Regul Integr Comp Physiol 286: R451-R464, 2004.

33. Peng YJ, Wang N, Gong QL, Li P. Caudal ventrolateral medulla mediates the depressor response elicited by the greater splanchnic nerve afferent stimulation in rats. Neuorsci Lett 325: 134-138, 2002.

34. Perrin J, Crousillat J. Splanchnic afferent input to the lateral reticular nucleus of the cat. J Auton Nerv Syst 8: 383-393, 1983.

35. Phillips SW, Gebber GL, Barman SM. Medullary lateral tegmental field: Control of respiratory rate and vagal lung inflation afferent influences on sympathetic nerve discharge. Am J Physiol Regul Integr Comp Physiol 288: R1396-R1410, 2005.

36. Pilowsky PM, Goodchild AK. Baroreceptor reflex pathways and neurotransmitters: 10 years on. J Hypertens 20: 1675-1688, 2002.

37. Pittam BS, Ewart WR, Appia F, Wingate DL. Physiological enteric stimulation elicits cardiovascular reflexes in the rat. Am J Physiol Gastrointest Liver Physiol 255: G319-G328, 1988.

38. Sato A, Schmidt RF. Somatosympathetic reflexes: Afferent fibers, central pathways, and discharge characteristics. Physiol Rev 53: 916-947, 1973. 
39. Saper CB. The central autonomic nervous system: Conscious visceral perception and autonomic pattern generation. Аппи Rev Neurosci 25: 433-469, 2002.

40. Sculcyzk P, Wilk M. Neural organization of the viscera-cardiac reflexes. Brain Res 326: 261-271, 1985.

41. Tjen-ALooi SC, Bonham AC, Longhurst JC. Interactions between sympathetic and vagal cardiac afferents in nucleus tractus solitarii. Am J Physiol Heart Circ Physiol 272: H2843-H2851, 1997.

42. Tjen-ALooi SC, Li P, Longhurst JC. Prolonged inhibition of rostral ventral lateral medullary premotor sympathetic neurons by electroacupuncture in cats. Auton Neurosci 106: 119-131, 2003.
43. Wang WZ, Gao ZL, Pan YX, Zucker IH, Wang W. Differential effects of cardiac sympathetic afferent stimulation on neurons in the nucleus tractus solitarius. Neurosci Lett 409: 146-150, 2006.

44. Weaver LC, Meckler RL, Fry HK, Donoghue S. Widespread neural excitation initiated from cardiac spinal afferent nerves. Am J Physiol Regul Integr Comp Physiol 245: R241-R250, 1983.

45. Zhou W, Fu LW, Tjen-ALooi SC, Guo ZL, Longhurst JC. Role of glutamate in a visceral sympathoexcitatory reflex in rostral ventrolateral medulla of cats. Am J Physiol Heart Circ Physiol 291: H1309-H1318, 2006.

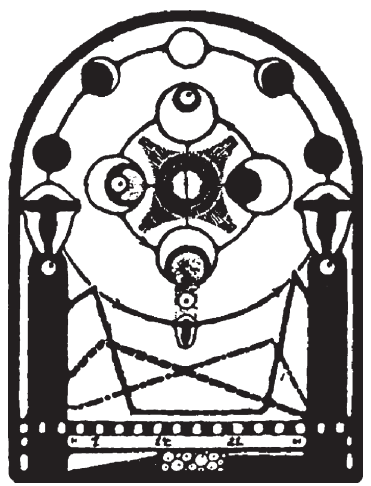

